\title{
Proceso de Bolonia (V): el currículo oculto
}

\author{
Felipe Rodríguez de Castro
}

\section{Introducción}

La educación médica es algo más que la mera transmisión de conocimientos y habilidades. De una forma consciente o inconsciente, los educadores también trasladan a los futuros médicos normas y valores que a menudo socavan los mensajes formales contemplados en los programas de formación o en el ideario de los centros sanitarios. De hecho, gran parte de la socialización secundaria ligada al proceso transformacional que supone convertirse en médico se produce fuera de los espacios considerados formalmente como docentes, lo que no le resta importancia en relación al conocimiento adquirido en situaciones más explícitas o formales [1]. Cada palabra pronunciada, cada acto ejecutado u omitido, cada broma gastada, cada silencio mantenido o cada contrariedad manifestada en un pasillo, en la cafetería o en el ascensor, tiene una influencia en el que aprende a nuestro lado mayor de lo que hubiéramos pretendido [2]. Quizá sea ésta una de las razones de por qué los cambios que las diversas reformas introducen en los currículos formales no siempre consiguen los resultados esperados.

En los últimos años se ha puesto de manifiesto que la buena práctica asistencial, además de las habilidades técnicas, necesita otro tipo de capacidades. Estas habilidades no técnicas (non-technical skills) forman parte de las conductas relacionadas con el desempeño profesional, que está condicionado, en gran medida, por un conjunto de premisas, no siempre fáciles de reconocer, las cuales constituyen la base de la cultura que se promulga en nuestros centros formativos. Este conjunto de premisas va a tener una profundísima influencia en los estudiantes y en los médicos en formación porque determina asunciones básicas acerca de lo que es 'aceptable' o 'inaceptable' en la práctica médica.

\section{¿Qué es el currículo oculto?}

El currículo oculto representa un trasfondo de normas, valores y reglas latentes en el proceso formativo, que los estudiantes han de asumir y adoptar para desempeñar convincentemente un papel social [3]. Es evidente que no todo lo que se enseña en la facultad o durante la residencia está contemplado en los programas. En realidad, una gran parte de lo que se enseña -y desde luego mucho de lo que se aprendetiene lugar fuera de los cursos o explicaciones formales. Pero el currículo oculto no es algo que se encuentre por casualidad, sino que se debe buscar intencionadamente [4]. Desde un punto de vista práctico, se podría definir como el conjunto de 'reglas, regulaciones y rutinas' institucionales no escritas [5]. Aunque el aprendizaje formal es importante, es el currículo oculto el que proporciona los elementos que permiten comprender las influencias existentes en el comportamiento del estudiante [6]. La mayor potencialidad educativa se consigue cuando ambos currículos, formal e informal, están alineados, lo que lamentablemente no siempre ocurre.

Reconocer que la educación médica incluye aspectos latentes o implícitos permite apreciar cuánto de lo que los estudiantes y residentes llegan a 'conocer' se adquiere o se fundamenta en interacciones que tienen lugar fuera de las aulas, de los laboratorios o, en general, al margen de los escenarios educativos tradicionales. Esta idea supone considerar los hospitales, los centros de salud y las instituciones formativas como entidades culturales y comunidades morales, íntimamente involucradas en la construcción de definiciones de lo que está bien y lo que está mal, lo que es 'buena' o 'mala' medicina. Además, y lo que es más importante, este concepto de currículo informal o currículo oculto exige de los profesores, de los tutores y de los educadores en
Facultad de Ciencias de la Salud. Universidad de Las Palmas de Gran Canaria. Las Palmas de Gran Canaria, España.

Correspondencia:

Dr. Felipe Rodríguez de Castro. Facultad de Ciencias de la Salud. Universidad de Las Palmas de Gran Canaria. Campus Universitario de San Cristóbal. Blas Cabrera Felipe, s/n. E-35016 Las Palmas de Gran Canaria.

E-mail:

dec_ccs@ulpgc.es

Conflicto de intereses: No declarado.

Competing interests: None declared.

(c) 2012 Educación Médica 
su conjunto el reconocimiento de la educación médica como un proceso cultural y, por tanto, como algo en constante cambio y sometido a fuerzas externas [7].

\section{Condicionantes externos del currículo oculto}

Estas continuas transformaciones a las que está sujeta la educación médica no sólo se relacionan con la evolución tecnológica y social, o los avances médicos en general, sino también con el perfil demográfico y la creciente diversidad socioeconómica, cultural y étnica de estudiantes y residentes [8]. En el año 2010, casi la mitad de los aspirantes a ocupar una plaza de médico especialista en formación en España era extranjero. Los extracomunitarios que compiten por las plazas de formación especializada han aumentado su presencia en la convocatoria de 2012 en un 15\% [9]. Además, en los centros formativos coexisten diferentes generaciones de médicos, con distintos valores y diferentes estilos de aprender y de trabajar. La personalidad y las características de la generación actual de estudiantes y residentes vienen condicionadas por el hecho de haber crecido en un mundo saturado de imágenes, ordenadores personales, Internet y nuevas formas de comunicación interpersonal. Los especialistas en formación se han educado en un mundo en el que se han producido cambios tecnológicos rápidos y profundos, y están acostumbrados al acceso a la información de una forma inmediata, ilimitada y sin esfuerzo. Como consecuencia de ello, son críticos con la organización y los métodos docentes jerárquicos tradicionales. Se sienten cómodos estableciendo relaciones con colegas que sólo conocen de forma virtual, lo que les permite organizarse sin restricciones geográficas para resolver problemas complejos.

Uno de los cambios demográficos más notables que se ha producido en los últimos años es el incremento de la proporción de mujeres en las últimas generaciones de médicos. Actualmente, suponen en torno al $40 \%$ de los médicos colegiados en España y hasta dos tercios de los estudiantes de medicina. En 1982, la cuarta parte de los profesores universitarios de ciencias de la salud eran mujeres, mientras que hoy día suponen casi la mitad. Este cambio no sólo es evidente en nuestro país, sino que también se constata en otras naciones de nuestro entorno geográfico y cultural. En Gran Bretaña se estima que las mujeres serán mayoría en el $\mathrm{Na-}$ tional Health System en el plazo de una década, y en Estados Unidos, en los últimos 20 años, la pro- porción femenina entre los estudiantes de medicina ha pasado de ser menor del $10 \%$ a alcanzar el $50 \%$. Este cambio en la demografía médica sin duda determina cambios en la cultura predominante en nuestros centros educativos. Sin pretender ser exhaustivo en este punto, las mujeres tienden a escoger especialidades menos tecnológicas, más orientadas al paciente y con trabajo más planificable. Este patrón, que se reproduce en la mayoría de los países occidentales, coincide con el creciente deseo de una mayor proporción de médicos -hombres y mujeres- de disponer de un trabajo más flexible y del diseño de carreras menos lineales, con períodos sabáticos o interrupciones ocasionales [10]. Todo esto implica una transformación en la concepción de algunos aspectos que, en relación con la dedicación al ejercicio de la profesión, han tenido generaciones previas.

\section{Algunas premisas latentes en educación médica}

Aprender a ser médico consiste en adoptar las normas de la profesión, lo que supone un proceso que tiene lugar en situaciones concretas y contextualizadas, e incluye, entre otros aspectos, la asimilación del impacto social que tiene la conducta de los miembros más influyentes del entorno [11]. Los estudiantes y los residentes tienden a internalizar los valores predominantes de la comunidad de práctica $y$, en este sentido, la educación médica debe entenderse como un proceso transformacional de identidades que precisa ajustes en la aplicación de unos principios idealizados y teóricos a la complejidad de los contextos reales. El currículo oculto funciona como un mecanismo de socialización dentro de una estructura educativa institucional más amplia que enseña a los estudiantes las dinámicas de poder que existen entre ellos y los legos, y que se fundamentan en la asimetría del conocimiento. En los hospitales, y en general en los centros educativos, existe un conjunto de asunciones y una serie de premisas que subyacen en ellas de forma implícita. Estas premisas no se reconocen fácilmente; son, más bien, una especie de 'ruido de fondo' cuya identificación es clave porque constituye el núcleo de normas no escritas que se transmiten, voluntaria o involuntariamente, en nuestras instituciones formativas, y que enmarcan las asunciones que los discentes finalmente aceptan como inevitables. Cambiar estas premisas se convierte, por consiguiente, en una tarea esencial para aquellos que intentan modificar la cultura médica vigente. 
La influencia del currículo oculto puede analizarse en múltiples aspectos y desde diferentes perspectivas. Sin pretender agotar todas las situaciones potenciales o los contextos clínicos o educativos, a continuación se analizan algunas de estas premisas latentes que participan en el proceso de aculturación profesional [12].

\section{Incertidumbre y complejidad}

En el ejercicio de la medicina no es frecuente admitir que algo se ignora o se desconoce. De hecho, siempre parece existir una explicación plausible o una respuesta correcta para cualquier problema médico, ya sea de naturaleza técnica o ética [13]. Esta actitud, de alguna forma, se arrastra desde las facultades de medicina, donde tanto profesores como alumnos se desenvuelven más confortablemente en el ámbito de la 'respuesta correcta'. En lugar de aprender a manejar la incertidumbre y el conocimiento probabilístico que caracterizan el ejercicio clínico, no es raro que una y otro se rechacen vehementemente tratando de transmitir a los pacientes y a los discentes una seguridad que, en muchas ocasiones, ni se tiene ni es posible. Además, como ha señalado recientemente Hilliard Jason en un MedEdWorld Webinars, la humildad del profesor es un instrumento vital, aunque frecuentemente menospreciado, para una educación médica efectiva. Se debe redescubrir y enseñar el valor del juicio clínico y la importancia de la reflexión personal, intelectual, científica y administrativa, en la toma de decisiones en la práctica médica [14].

La naturaleza de los dilemas morales a los que se enfrenta un estudiante o un residente es una fuente constante de incertidumbre y ansiedad que no siempre se maneja de forma adecuada. La mayoría de las intervenciones educativas que se realizan con objeto de desarrollar la formación ética de los futuros médicos se centra en aspectos relacionados con el ejercicio profesional. Este abordaje, sin duda necesario, soslaya los conflictos éticos a los que se enfrenta el estudiante como tal y para los cuales no recibe ningún apoyo u orientación específica [15]. En la mayoría de los casos, los estudiantes son abandonados sin ofrecerles la oportunidad de discutir o de compartir estos conflictos. El residente también afronta otra clase de problemas éticos inherentes al proceso formativo en sí mismo. Estos dilemas surgen por las obligaciones éticas que tiene como médico y como estudiante, como médico y tutelado, y como médico y empleado de una institución docente [16]. Estas lealtades encontradas pueden ser éticamente difíciles de resolver para médicos forma- dos, pero para un residente, la negociación de estos dilemas en un momento de alto nivel de estrés, cuando las debilidades personales -miedo, cansancio, dudas, ignorancia- conducen, en ocasiones, a tomar decisiones éticamente dudosas, la resolución de estos conflictos es particularmente compleja. Aquellos que no tengan recursos personales o sociales para resolver estos dilemas optarán por la solución más sencilla: 'seguir la corriente' y obedecer las normas y las reglas explícitas o implícitas. Hafferty y Franks sostienen que los estudiantes de medicina sufren la inseguridad profesional y el miedo a la equivocación también como una incompetencia ética [17]. En este sentido, las normas y los valores que se transmiten en el currículo oculto se pueden percibir como moralmente aceptables al carecer de referencias propias.

\section{Infalibilidad del médico}

Este aserto latente contrasta con datos de la Organización Mundial de la Salud (OMS) según los cuales el 10\% de los pacientes hospitalizados sufren un suceso adverso que les supone algún daño o una prolongación de su estancia. A pesar de este elevado porcentaje, es excepcional que en una sesión general de nuestros centros educativos se debata abiertamente acerca de equivocaciones o errores cometidos durante el proceso de diagnóstico, tratamiento o seguimiento de los pacientes, y sobre la forma de evitarlos en el futuro. Se calcula que en Estados Unidos se produce una intervención quirúrgica en el lugar erróneo o en un paciente equivocado cada 75.000 operaciones [18]. En nuestro país, en una encuesta sobre errores profesionales dada a conocer recientemente, se constata que el $76 \%$ de los médicos reconoce que se ha equivocado alguna vez -aunque le cuesta concretar en qué consistió el error-y sólo el $40 \%$ admite habérselo comunicado al paciente. Esta situación no deja de ser paradójica dado que las denuncias médicas suelen ser producto de la combinación de un error y de la falta de información al paciente respecto a ello. En cualquier caso, es interesante constatar el modo tan diverso en el que los médicos comunican sus errores a los pacientes y la manera en que este proceso está cambiando [19].

\section{Entornos intimidatorios}

Lo más dramático es que la mayoría de los errores se podría haber evitado fácilmente. En muchos casos, la única causa atribuida a la equivocación es, sencillamente, la falta de comunicación entre los 
miembros del equipo asistencial. Sin duda, la solución para evitar los errores médicos no es tan simple como repasar una lista de tareas. Es preciso un cambio cultural porque, en muchas ocasiones, el ambiente laboral que se respira es muy intimidatorio y jerarquizado [20]. De hecho, no es raro que en nuestros centros sanitarios se justifiquen comportamientos inapropiados o groseros por la importancia de la tarea que se está desarrollando o la delicada situación que se está viviendo, subrayando de esta manera la importancia del resultado por encima del proceso. En este sentido, estudiantes y residentes se quejan de frecuentes actitudes de intimidación, reprobación pública y humillación -un abuso del método socrático-, que inducen a esconder lo que se ignora y que estimulan un entorno donde se tiene miedo a preguntar y a aclarar dudas o aspectos de difícil comprensión [21]. Un artículo reciente evaluó el nivel de menosprecio y de acoso sufrido por más de 1.500 estudiantes de 16 facultades de medicina estadounidenses. Al final del último año, el $42 \%$ manifestaba haber sufrido algún tipo de acoso y el $84 \%$ pensaba que había sido menospreciado en algún momento [22]. Uno de los aspectos más llamativos de este trabajo es que los principales responsables de estos comportamientos reprobables son los residentes o los profesores de asignaturas clínicas, que se supone que deben servir de modelo a imitar para la nueva generación de médicos. En Canadá, también se ha comunicado abuso psicológico en hasta el $93 \%$ de los médicos residentes [23]. El humor cínico o despectivo por parte del personal sanitario -que frecuentemente es disculpado como una forma de sobrellevar el estrés, el cansancio y las dificultades emocionales intrínsecas de las profesiones sanitarias- también debe considerarse un abuso verbal, una falta de respeto y una forma de deshumanización de los pacientes y de los discentes que lo sufren y de los docentes que lo cultivan [24].

Los distintos escenarios en los que se produce la asistencia clínica deberían ser lugares idóneos para transferir los valores profesionales y donde los tutores y docentes pudieran ejercer su función de 'modelo a imitar' de forma eficaz. Sin embargo, el entorno clínico no siempre constituye un clima ideal para el aprendizaje [25]. Los estudiantes y los residentes tienden a interiorizar los valores de la comunidad donde se están formando, pero cuando en estos espacios educativos no hay correspondencia entre la prédica y la práctica, el discente percibe que su formación se basa en inconsistencias, contradicciones y mensajes ambivalentes, lo que abocará inevitablemente a un comportamiento cínico y a un relativismo moral. Hafferty [7] define el currículo oculto precisamente como el impacto que causa en quienes aprenden el hecho de ver cómo se están haciendo las cosas, especialmente si no coincide con el discurso de cómo deberían hacerse. Por consiguiente, como señalan Prat et al [26], habrá que cuidar los comportamientos docentes en los entornos formativos para no arruinar el entusiasmo de los que se inician en el aprendizaje de la profesión. De hecho, se ha documentado repetidamente en la bibliografía [27-29] que, a medida que el estudiante de medicina avanza en su proceso formativo, se produce una serie de transformaciones no siempre positivas. Los estudiantes llegan a la facultad con una actitud idealizada y un tanto ingenua que muy pronto comienza a marchitarse dejando patentes actitudes cínicas, arrogantes y de desapego emocional. En un sistema fuertemente jerarquizado, los estudiantes perciben que, para progresar, la solución menos dolorosa es la adhesión incondicional a las reglas y normas establecidas [30,31]. Hren et al constatan la regresión en el razonamiento moral de los estudiantes de medicina, que se manifiesta como una convergencia hacia un esquema basado en el 'mantenimiento de las normas' [32]. Feudtner et al [28] describen que un $98 \%$ de los estudiantes entrevistados en su estudio fue testigo de una actitud peyorativa del médico hacia el paciente, el $61 \%$ observó lo que a su juicio fue un comportamiento poco ético por parte de algún otro miembro del equipo asistencial, el $67 \%$ se sintió incómodo o culpable por algo que había hecho como interno y el $62 \%$ reconocía que sus principios éticos se habían deteriorado gravemente. Así las cosas, lo que detectamos entre nuestros estudiantes y residentes es un malestar psicológico, un estrés y una angustia cada vez mayores. Son personas que llegan con expedientes académicos impolutos y con altas expectativas personales que la vida diaria se va encargando de desgastar. Por tanto, no es extraño comprobar que, según un estudio de la Fundación Galatea, el 30\% de los médicos internos residentes de nuestro país padece malestar psicológico por su desempeño laboral, lo que supone una incidencia tres veces superior a la observada en el resto de la población de la misma edad. Esta situación está desencadenando un mayor abuso de sustancias, trastornos alimentarios, cinismo y baja empatía entre nuestros jóvenes colegas. En definitiva, algunos médicos se convierten en verdaderos agentes tóxicos porque enseñan o muestran conductas ajenas al profesionalismo médico; en este mismo sentido, profesores y tutores tienen un papel potencial muy relevante como agentes de salud. 
El aprendizaje se construye y, por consiguiente, los médicos que se están formando no reciben información, sino que se exponen a ideas sobre las que construirán un significado, conectarán con experiencias y conocimientos previos, evaluarán y sobre las que elaborarán juicios. En este paradigma, las relaciones tutor-residente o profesor-estudiante y el entorno educativo son importantes porque proporcionan el contexto que da forma a la construcción del aprendizaje. Una buena relación residentetutor o una cultura institucional abierta se asocia con una motivación intrínseca para el aprendizaje. Un contexto de emociones negativas, como el miedo o la ansiedad, puede interferir con este proceso porque altera la capacidad del aprendiz para procesar la información de una manera eficiente. De la misma manera, las emociones positivas disminuyen la ansiedad y llevan al estudiante o al médico en formación a adoptar comportamientos positivos de aprendizaje, como hacer preguntas, asumir retos o probar hipótesis [33].

\section{Atención centrada en el paciente}

El currículo formal hace hincapié en la prevalencia de los intereses del paciente por encima de cualquier otra consideración. Nadie pone en duda la pertinencia de esta actitud, si bien, en la práctica clínica, cuesta concretar qué significa exactamente centrar la atención en el paciente. Por el contrario, lo que sabemos es que los médicos continúan sin poder o sin querer dar a sus pacientes el tiempo suficiente para discutir sus problemas, sus miedos y sus preocupaciones. En algunos estudios se ha observado que los médicos interrumpen a sus pacientes a los 18 segundos de iniciar la explicación de sus síntomas, a pesar de que lo habitual es que los enfermos sólo hablen espontáneamente una media de 92 segundos. Las consultas son percibidas por el paciente como situaciones estresantes que tienen lugar en un ambiente hasta cierto punto hostil y, en consecuencia, recuerdan menos del $50 \%$ de lo que se les dice durante la entrevista clínica [34].

En el informe de la OMS del año 2000 se introdujo un concepto interesante que puede ayudar a definir la atención centrada en el paciente. Se trata del concepto de 'responsividad' (responsiveness). Los resultados en salud -supervivencia, por ejemplo- no miden aspectos no sanitarios, como las expectativas personales de cada paciente o el trato recibido. La idea de 'responsividad' se refiere a estos aspectos. Se podría decir, como sugiere Escarrabill, que mide el coste personal que tiene para el paciente la obtención de unos resultados clínicos determinados. En el informe de la OMS se identifican diversos elementos que configuran el concepto. Estos elementos se agrupan en dos grandes dimensiones: respeto a las personas y orientación al cliente. Los centros sanitarios son responsables de las políticas institucionales, de la cultura organizativa y de los códigos de conducta que reconoce -aunque sea de forma tácita- como buenas prácticas. Los tiempos asignados para atender a los pacientes, las explicaciones escritas que se les proporcionan acerca de su enfermedad y sus expectativas, la continuidad del mismo médico en la atención del enfermo, la estructura arquitectónica de los espacios asistenciales de forma que garantice la confidencialidad y la dignidad de las personas, la manera como se llama a los pacientes en las salas de espera, los rótulos de las consultas con identificaciones demasiado explícitas acerca de la enfermedad que se sufre, los tiempos de espera con inaceptables cargas de sufrimiento e incertidumbre, etc., configuran la cultura institucional y el código de conducta que ésta promulga.

La atención centrada en el paciente implica también un cambio de actitud en el que se acepte que el núcleo de la toma de decisiones no radica exclusivamente en el médico [35]. Cada vez es más frecuente que el paciente se implique en la elección del tratamiento o en la decisión de cómo abordar su problema de salud. Esta participación del paciente se relaciona directamente con la cultura médica predominante. Si ésta es paternalista, tanto el médico como el paciente asumirán que la decisión es responsabilidad exclusiva del primero. En un modelo cultural más igualitario, se suele optar por una fórmula de responsabilidad compartida [36]. La relación clínica es una relación de deliberación y este concepto no siempre es bien entendido. Todo diálogo constituye una deliberación y la deliberación es un procedimiento técnico que tiene su complejidad y que requiere unos hábitos que hay que adquirir y que no siempre son fáciles de aprender.

\section{Trabajo en equipo}

La deliberación fundamental para tomar decisiones es colectiva y deliberar colectivamente es difícil porque al poner en común posturas, criterios u opiniones, los otros pueden discrepar o quitarnos la razón, y eso no suele recibirse de buen grado. Las rutinas sanitarias se basan en equipos interdisciplinarios, en la colaboración profesional y en la toma de decisiones en grupo, por lo que una interacción entre distintos profesionales sanitarios resulta crucial para garantizar un servicio asistencial de cali- 
dad y enfocado a la atención social [37]. Todos los profesionales de la salud deberían ser formados para utilizar conocimientos, involucrarse en el razonamiento crítico y seguir una conducta ética, de manera que sean capaces de participar en sistemas de salud centrados en el paciente y en la población, como miembros de equipos sensibles, receptivos y conectados de forma global. Los médicos no debemos insistir en que somos nosotros los únicos que tomamos siempre todas las decisiones. Hay que 'empoderar' a los otros miembros del equipo porque de esta manera ganamos respeto entre nuestros pares y subordinados y, sobre todo, creamos una cultura de lealtad (38). La jerarquía top-down predominante hace dos o tres décadas está pasada de moda y, además, puede llegar a ser peligrosa. Como han observado algunos autores, existe una relación directa entre un comportamiento arrogante, intimidatorio, rudo o grosero en el quirófano y la mortalidad o la tasa de complicaciones postoperatorias. Conductas de estas características por parte del cirujano tienen, como primera consecuencia, una mayor rotación del personal de enfermería y un incremento del número de errores. En 2002, un estudio realizado en hospitales de la Veterans Administration [39] mostró que el 96\% de las enfermeras había sido testigo de algún comportamiento reprobable por parte del médico. Diez años antes, Díaz y McMillin [40] comunicaron que el $64 \%$ de las enfermeras había sufrido abuso verbal por parte de algún médico al menos una vez cada dos o tres meses, y que el 23\% de ellas había sido amenazada físicamente. Este tipo de situaciones puede tener consecuencias nefastas para los pacientes porque la mayoría de las enfermeras, ante unas órdenes médicas confusas, asumen que el tratamiento prescrito es seguro o correcto con tal de evitar la consulta con médicos concretos que han tenido este tipo de comportamiento negativo.

\section{Democratización del conocimiento y cambios tecnológicos}

En la actualidad, con el acceso a la información que proporcionan las nuevas tecnologías, estudiantes, residentes o incluso los propios pacientes pueden disponer de tanta información como un médico experimentado. La información ya no fluye, como se comentaba antes, de arriba hacia abajo, sino que tiene un curso bidireccional. Varios autores han apuntado el poder de Google en la práctica clínica, sugiriendo que puede proporcionar un diagnóstico correcto en más del $50 \%$ de los casos clinicopatológicos que publica periódicamente The New England
Journal of Medicine [41]. De hecho, en la actualidad, hay más búsquedas científicas que se inician con Google que con PubMed [42]. Todo ello demuestra que la disponibilidad inmediata de una información prácticamente ilimitada tiene un enorme potencial transformador, no sólo del conocimiento de los residentes o estudiantes, sino también de sus relaciones con sus tutores, sus colegas y sus pacientes. Todo el mundo tendrá que adaptarse a esta nueva situación, pero, como en todas las revoluciones, los que tendrán que hacer un mayor esfuerzo serán aquellos que están situados en lo más alto de la pirámide jerárquica en el momento actual. Se ha sugerido, además, que la competencia declina con el tiempo tras finalizar el período de formación y que hay una relación inversa entre el tiempo que se lleva ejerciendo y la calidad de la asistencia que se presta [43]. Probablemente, la pendiente de caída de la competencia viene condicionada por la aceleración de los cambios científicos y tecnológicos. En el futuro más inmediato, posiblemente cada generación ofrecerá habilidades complementarias que contribuyan a la formación del resto del equipo con el objeto de mejorar la calidad de la asistencia a los pacientes. En todo caso, sí parece claro que, como subraya Margaret Mead, estamos viviendo la brecha generacional mayor de la historia y nos adentramos en una época en que los jóvenes ya no tienen como modelo de conducta a sus padres, sino a personas de su propia generación. Los miembros de la llamada 'generación Y' han comprobado que algunos de sus integrantes han alcanzado posiciones sociales y económicas destacadas, lo que condiciona que el sistema tradicional de jerarquías sea visto con cierta indiferencia. Tal vez por esto, una de las características que más valoran los residentes en los médicos con los que se relacionan es que sean jóvenes [44]. Sea como fuere, lo cierto es que un número significativo de tutores siente que sus residentes están más preocupados que ellos por armonizar su vida personal con la laboral y por su calidad de vida, y menos dispuestos al compromiso institucional y al trabajo sin límite de horario que caracterizó a su generación. En cualquier caso, en el momento actual se dispone de muy pocos datos acerca de la actitud laboral de los médicos más jóvenes [45].

\section{Investigación}

La investigación también ocupa un lugar preferente en el currículo oculto y ofrece otra interesante oportunidad a los educadores para formar en valores. Sin embargo, desgraciadamente, en este campo tam- 
poco las noticias son buenas. Según Titus y Bosch, el $2 \%$ de los investigadores reconoce haber tenido comportamientos científicos inadecuados al menos una vez, lo que en términos absolutos supone unos 8.000 investigadores en Estados Unidos. Hasta el $34 \%$ de los científicos admite una o más prácticas investigadoras cuestionables, como análisis inapropiados, exageración deliberada de los hallazgos y cambios en el diseño del estudio [46]. Además, sólo el $24 \%$ de los investigadores que fueron testigos de comportamientos científicos inapropiados, lo comunicó a sus superiores. Los tutores deben enseñar conductas responsables en investigación y las instituciones tienen que establecer una cultura de la honradez y del comportamiento responsable. La veracidad en las autorías, los conflictos de intereses, el manejo de datos, etc., son sólo algunos ejemplos de lo que se ha comenzado a denominar un 'comportamiento institucional responsable'. La tarea se ha complicado progresivamente, sobre todo si tenemos en cuenta que para la generación del milenio -aquellos que ingresaron en la universidad en el año 2000-, compartir es un elemento clave en su vida y esta socialización les ha enseñado a 'cortar, pegar, copiar, bajar.., tal vez con una excesiva desenvoltura. Twenge observó un incremento persistente en el porcentaje de estudiantes de últimos años de bachillerato que admitían haber engañado deliberadamente en sus exámenes, pasando de un $34 \%$ en 1969 , a un $61 \%$ en 1992 y un $74 \%$ en el año 2002 [47].

\section{Gestión y relaciones con la industria}

El carácter especializado del conocimiento de los profesionales sitúa a los médicos como agentes de dos principales: los pacientes y los gestores de los centros. La dependencia de más de un principal, junto a la difícil cuantificación de los resultados de los servicios médicos, la urgencia y la sobrecarga de trabajo, favorece un trasvase de la autoridad real (derecho efectivo de decisión y control) desde quienes ostentan la autoridad formal (directivos y administradores) hacia los médicos. Estos últimos, a falta de esquemas de incentivos apropiados, podrían inducir al consumo excesivo. Pero la gestión de los recursos es también un problema moral [48]. Una parte considerable, aunque no muy bien medida, del gasto sanitario es innecesaria: prescripciones de medicamentos inadecuadas, estancias e ingresos hospitalarios inapropiados o efectividad marginal de muchas pruebas e intervenciones diagnósticas. Esta proporción de actividad sanitaria improcedente puede ser consecuencia de un déficit de formación, pero también puede tener su raíz en la práctica de una medicina defensiva, en la personalidad y en la forma en que cada médico ejerce la profesión, el modo en que maneja la incertidumbre y los diferentes grados de aversión al riesgo y la prudencia. Estos rasgos de la personalidad del médico marcan diferencias en sus hábitos de prescripción o indicación de exploraciones [49] y condicionan una determinada cultura institucional. Algunos autores han observado que los médicos que más precozmente prescriben nuevos medicamentos tienen un perfil determinado: son jóvenes y formados en determinadas facultades [50].

La separación entre factores de oferta y demanda no siempre está clara en un mercado imperfecto como el sanitario, donde la demanda está, al menos en parte, inducida por la oferta. Habitualmente es el médico quien determina la demanda que los pacientes harán de los servicios sanitarios. Se establece una relación de agencia en la que existen incentivos para elevar el gasto. Un ejemplo claro es la industria farmacéutica, que además de producir soluciones a necesidades, genera necesidades y multiplica el número de enfermos con el desarrollo del llamado 'marketing del malestar' [51]. Aunque los conflictos de intereses están generalizados en la vida profesional del médico, su formación en cualquiera de sus etapas no suele contemplar la enseñanza de cómo abordar esos conflictos [52]. Se ha propuesto un análisis lógico como herramienta nuclear en el manejo de estos dilemas. Por ejemplo, para alcanzar un objetivo (A: ser un médico ético e informado que ejerce la medicina basada en la mejor evidencia disponible), debe satisfacerse una condición (B: estar actualizado y disponer de la información clínica más reciente). Esta condición se consigue mediante una acción (D: acudir a cursos de formación continuada esponsorizados por la industria farmacéutica). Pero, alternativamente, conseguir el objetivo A requiere otra condición (C: disponer de información objetiva y tener libertad para actuar en base a ella), lo que se logra mediante la acción D' (evitando participar en actividades de formación subvencionadas por la industria). La exposición de objetivos, necesidades, deseos y asunciones permite modificar una o más de estas variables para resolver el conflicto, tal vez sustituyendo una de las condiciones necesarias $(\mathrm{B}, \mathrm{C})$ o de las acciones (D, D'), o incluso encontrando la manera de llevar a cabo ambas, D y D.' Este tipo de análisis puede ayudar a los clínicos a identificar y resolver conflictos y ser de mayor utilidad que mantener de forma simplista el aserto: 'no acepte regalos o ayudas porque no es profesional' [53]. 


\section{Prestigio de la especialidad}

Desde hace años se habla del déficit de médicos en determinadas especialidades. Entre estas especialidades deficitarias siempre figura la de medicina familiar y comunitaria que, de manera paradójica, es relativamente poco demandada por los egresados de nuestras facultades. Es posible que comentarios peyorativos que escuchan nuestros estudiantes en los centros formativos en relación a esta disciplina formen parte del currículo oculto, lo que junto con el prestigio actual de la tecnología, las expectativas de realización y mejora profesional y de las condiciones laborales, así como la posibilidad de ejercicio de la medicina privada, expliquen parcialmente esta situación. Sea como fuere, lo cierto es que este estado de cosas no es privativo de nuestro país y algunos investigadores se han preocupado de demostrar lo injustificado de este aspecto latente en la formación de nuestros médicos [54].

\section{Evolución del profesionalismo}

Como señala Oriol, reconocer que la medicina actual tiene una base científico-técnica no implica entender la medicina exclusivamente como una ocupación para técnicos de alta cualificación. La medicina trata con pacientes, algunos de los cuales pueden ser tratados con éxito, pero también se responsabiliza del cuidado de aquellas otras personas -hoy día mayoritarias- cuyas dolencias sólo pueden paliarse. La medicina es una actividad moral cuya función primordial es sanar, lo que depende en gran medida de la confianza que el paciente y la sociedad depositan en ella. El profesionalismo es el conjunto de valores y compromisos por los cuales la sociedad otorga a los médicos un contrato social específico y los reconoce como profesión [55].

La educación médica ha promocionado la importancia y la necesidad de formar a los estudiantes en el profesionalismo [56,57], que contiene valores como la honradez, la integridad, la primacía del bienestar del paciente y su autonomía, la compasión, la empatía, el altruismo, el comportamiento ético y la justicia social [58]. Estos atributos están altamente considerados por los educadores, de forma que un número creciente de facultades de medicina de todo el mundo ha diseñado cursos, seminarios o asignaturas específicamente orientados a conseguir que estos valores arraiguen en el ejercicio profesional de los futuros médicos [56,59]. Esta noción de profesionalismo parece entrar en conflicto frecuente con muchas de las premisas laten- tes en el currículo. De hecho, a pesar de la constante repetición del mantra de los valores humanísticos, de las habilidades en la comunicación efectiva, de la conectividad con el paciente, del comportamiento coherente y positivo, etc., estas cualidades no se reflejan de una manera sistemática en los procesos formativos [60]. Algunos autores han establecido una clara relación entre las experiencias vividas por el discente en el currículo oculto y aspectos particulares del profesionalismo, los contextos en los que emergen estas experiencias críticas [61], o formas más explícitas alternativas al proceso de socialización espontánea -dependientes de los modelos profesionales a los que se encuentra expuesto el que aprende y a la cultura institucional predominante del centro educativo-, como forma de alcanzar competencias relacionadas con el profesionalismo [62].

No está claro cómo influirán los cambios generacionales, demográficos y tecnológicos en la filosofía educativa y en la visión del profesionalismo. Éste no es estático, sino que evoluciona con su contexto social, aunque resida sobre un núcleo de valores permanentes. Es posible que la nueva generación de médicos no acepte fácilmente que otros les digan cuáles deben ser sus valores, sus principios y sus compromisos. Está bien que así sea, pero algunos datos son preocupantes. En los últimos 25 años, el Narcissistic Personality Inventory Score -índice que mide el nivel de narcisismo- ha aumentado en dos tercios de los escolares estadounidenses. Ha habido un $30 \%$ de incremento desde 1982, que es cuando se aplicó por primera vez este índice [63]. La importancia de estos hallazgos radica en que el narcisismo, en su forma más extrema, hay que contemplarlo como una personalidad en la cual el engaño, la falta de empatía y de sensibilidad y el comportamiento egocéntrico son atributos dominantes.

Seguramente, el equilibrio entre la vida personal y profesional adquiera una creciente importancia. El altruismo y la justicia social permanecerán como principios básicos inmutables para la mayor parte de los médicos, pero la autoformación continuada, la atención a la familia y la flexibilidad en la carga de trabajo pueden emerger como valores. Coherentes con estos cambios evolutivos en la noción de profesionalismo, los jóvenes médicos pueden diseñar su carrera de forma muy diferente a la visión tradicional. Tal vez no se mantenga un compromiso de por vida con una especialidad en particular -sobre todo si la nueva organización troncal permite una mayor permeabilidad formativa-, lo que con toda seguridad supondrá nuevos retos y equilibrios entre valores e intereses, personales e institucionales. 
Quizá sea necesario abordar alguno de los aspectos latentes que se han mencionado de una manera más abierta y explícita, extrayéndolos de su ubicación 'oculta' dentro del currículo y analizar de qué modo están influyendo en la formación de nuestros estudiantes y residentes, o cómo están evolucionando.

\section{Bibliografía}

1. Lingard L, Reznick R, DeVito I, Espin S. Forming professional identities on the health care team: discursive constructions of the 'other' in the operating room. Med Educ 2002; 36: 728-34.

2. Mahood SC. Beware the hidden curriculum. Can Fam Physician 2011; 57: 983-5.

3. Wren DJ. School culture: exploring the hidden curriculum. Adolescence 1999; 34: 593-6.

4. Martin JR. What should we do with a hidden curriculum when we find one? In Martin JR, ed. Changing the educational landscape: philosophy, women, and curriculum. New York: Routledge; 1994. p. 154-69.

5. Bennett N, Lockyer J, Mann K, Batty H, Laforet K, Rethans JJ, et al. Hidden curriculum in continuing medical education. J Contin Educ Health Prof 2004; 24: 145-52.

6. Roberts JH, Sanders T, Mann K, Wass V. Institutional marginalisation and student resistance: barriers to learning about culture, race and ethnicity. Adv Health Sci Educ 2010, 15: 559-71.

7. Hafferty FW. Beyond curriculum reform: confronting medicine's hidden curriculum. Acad Med 1998; 73: 403-7.

8. Murray-García JL, García JA. The institutional context of multicultural education: what is your institutional curriculum? Acad Med 2008; 83: 646-52.

9. García-Sampedro H. MIR 2011: crece la demanda de unas plazas que disminuyen. Revista Médica 2012; 133: 16-20.

10. Winyard G. The future of female doctors. BMJ 2009; 338: 1397-8

11. Gual A, Palés J, Nolla M, Oriol A. Proceso de Bolonia (III). Educación en valores: profesionalismo. Educ Med 2011; 14: 73-81.

12. Oriol A. Bolonia y la aculturación profesional. Educ Med 2011; 14: 71-2.

13. Schrauth M, Kowalski A, Weyrich P, Begenau J, Zipfel S, Nikendei C. Self-image, the real physician and the ideal physician: a comparison of medical students from 1981 and 2006. Psychother Psychosom Med Psychol 2009; 59: 446-53.

14. Palfrey S. Daring to practice low-cost medicine in high-tech era. N Engl J Med 2011; 364: e21.

15. Christakis DA, Feudtner C. Ethics in a short white coat: the ethical dilemmas that medical students confront. Acad Med 1993; 68: 249-54.

16. Hoop JG. Hidden ethical dilemmas in psychiatric residency training: the psychiatry resident as dual agent. Acad Psychiatry 2004; 28: 183-9.

17. Hafferty FW, Franks R. The hidden curriculum, ethics teaching, and the structure of medical education. Acad Med 1994; 67: 861-71.

18. Stahel PF, Sabel AL, Victoroff MS, Varnell J, Lembitz A, Boyle DJ, et al. Wrong-site and wrong-patient procedures in the universal protocol era: analysis of a prospective database of physician self-reported occurrences. Arch Surg 2010; 145: 978-84.

19. Gallagher TH, Garbutt JM, Waterman AD, Flum DR, Larson EB, Waterman BM, et al. Choosing your words carefully. How physicians would disclose harmful medical errors to patients. Arch Intern Med 2006; 166: 1585-93.

20. Makary MA. The hazard of more reporting in quality measurement: comment on 'Wrong-site and wrong-patient procedures in the universal protocol era.' Arch Surg 2010; 145: 984.

21. Lempp H, Seale C. The hidden curriculum in undergraduate medical education: qualitative study of medical students' perceptions of teaching. BMJ 2004; 329: 770-3.

22. Frank E, Carrera JS, Stratton T, Bickel J, Nora LM. Experiences of belittlement and harassment and their correlates among medical students in the United States: longitudinal survey. BMJ 2006; 333: 682.

23. Cook DJ, Liutkus JF, Risdon CL, Griffith LE, Guyatt GH, Walter SD. Residents' experiences of abuse, discrimination and sexual harassment during residency training: McMaster University Residency Training Programs. CMAJ 1996; 154: 1657-65.

24. Berk R. Derogatory and cynical humour in clinical teaching and the workplace: the need for professionalism. Med Educ 2009; 43: 7-9.

25. Neumann M, Edelhäuser F, Tauschel D, Fischer MR, Wirtz M, Woopen C, et al. Empathy decline and its reasons: a systematic review of studies with medical students and residents. Acad Med 2011; 86: 996-1009.

26. Prat J, Palés J, Nolla M, Oriol A, Gual A. Proceso de Bolonia (II): educación centrada en el que aprende. Educ Med 2010; 13: 197-203.

27. Coulehan J, Williams PC. Vanquishing virtue: the impact of medical education. Acad Med 2001; 76: 598-605.

28. Feudtner C, Christakis DA, Christakis NA. Do clinical clerks suffer ethical erosion? Students' perceptions of their ethical environment and personal development. Acad Med 1994; 69: 670-9.

29. Baldwin DC Jr, Daugherty SR, Rowley BD. Unethical and unprofessional conduct observed by residents during their first year of training. Acad Med 1998; 73: 1195-200.

30. Morton KR. Why does moral reasoning plateau during medical school? Acad Med 1996; 71: 5-6.

31. Branch WT. Supporting the moral development of medical students. J Gen Intern Med 2000; 15: 503-8.

32. Hren D, Marusic M, Marusic A. Regression of moral reasoning during medical education: combined design study to evaluate the effect of clinical study years. Plos One 2011; 6: e17406.

33. Haidet P, Stein HF. The role of the student-teacher relationship in the formation of physicians. The hidden curriculum as process. J Gen Intern Med 2006; 21: S16-20.

34. Roberts NJ, Partridge MR. Optimal consultations for those with respiratory illness. Breathe 2007; 3: 331-7.

35. Berwick DM. What 'patient-centered' should mean: confessions of an extremist. A seasoned clinician and expert fears the loss of his humanity if he should become a patient. Health Affairs 2009; 28: 555-65.

36. Coulter A, Jenkinson C. European patients' views on the responsiveness of health systems and healthcare providers. Eur J Public Health 2005; 15: 355-60.

37. Frenk J, Chen L, Bhutta ZA, Cohen J, Crisp N, Evans T, et al. Health professionals for a new century: transforming education to strengthen health systems in an interdependent world. Lancet 2010; 376: 1923-58.

38. Klein AS, Forni PM. Barbers of civility. Arch Surg 2011; 146: 774-7.

39. Rosenstein AH. Nurse-physician relationships: impact on nurse satisfaction and retention. Am J Nurs 2002; 102: 26-34.

40. Díaz AL, McMillin JD. A definition and description of nurse abuse. West J Nurs Res 1991; 13: 97-109.

41. Tang $\mathrm{H}, \mathrm{Ng} \mathrm{JH}$. Googling for a diagnosis - use of Google as a diagnostic aid: internet-based study. BMJ 2006; 333: 1143-5.

42. Campion EW, Drazen JM. Revistas con revisión externa (peer review) en la era digital. Arch Bronconeumol 2010; 46: 569-70.

43. Choudhry NK, Fletcher RH, Soumerai SB. Systematic review: the relationship between clinical experience and quality of health care. Ann Intern Med 2005; 142: 260-73.

44. Vázquez G, Rodríguez M, Rucabado L, García A, Murillo F, Navarrete P, et al. Los residentes en las unidades de cuidados intensivos, ¿cuál es la percepción de la formación que reciben? Educ Med 2011; 14: 189-94.

45. Elkind MSV. Teaching the next generation of neurologists. Neurology 2009; 72: 657-63. 
46. Titus $\mathrm{S}$, Bosch $\mathrm{X}$. Tie funding to research integrity. Nature 2010; 466: 436-7.

47. Twenge JM. Generation Me: why today's young Americans are more confident, assertive, entitled -and more miserable than ever before. New York: Free Press; 2006.

48. Hafferty FW, Brennan M, Pawlina W. Professionalism, the invisible hand, and a necessary reconfiguration of medical education. Acad Med 2011; 86: e5.

49. Del Llano-Señarís JE. Gestión clínica y sanitaria: ayudando a conciliar necesidad y escasez. Gestión Clínica y Sanitaria 2003; 5: 3-6.

50. Tamblyn R, McLeod P, Hanley JA, Girard N, Hurley J. Physicians and practice characteristics associated with the early utilization of new prescription drugs. Med Care 2003; 41: 895-908

51. Moynihan R. The making of a disease: female sexual dysfunction. BMJ 2003; 326: 45-7.

52. Fitz MM, Homan D, Reddy S, Griffith CH 3rd, Baker E, Simpson KP. The hidden curriculum: medical students' changing opinions toward the pharmaceutical industry. Acad Med 2007; 82 (Suppl 10): S1-3.

53. Pauker SG, Wong JB. How (should) physicians think? A journey from behavioral economics to the bedside. JAMA 2010; 304: 1233-5.

54. Woloschuk W, Wright B, McLaughlin K. Debiasing the hidden curriculum. Can Fam Physician 2011; 57: e26-30.
55. Oriol A. El profesionalismo: asignatura pendiente del sistema educativo médico. Eidon 2010; 33: 52-7.

56. Baernstein A, Amies-Oelschlager A, Chang T, Wenrich M. Learning professionalism: perspectives of preclinical medical students. Acad Med 2009; 84: 574-81.

57. Elliott DD, May W, Schaff P, Nyquist JG, Trial J, Reilly JM, et al. Shaping professionalism in pre-clinical medical students: professionalism and the practice of medicine. Med Teach 2009; 31: e295-302.

58. Dyrbye LN, Massie FS Jr, Eacker A, Harper W, Power D, Durning SJ, et al. Relationship between burnout and professional conduct and attitudes among US medical students. JAMA 2010; 304: 1173-80.

59. Branch W. The road to professionalism: reflective practice and reflective learning. Patient Educ Couns 2010; 80: 327-32

60. Michalec B. Learning to cure, but learning to care? Adv Health Sci Educ Theory Pract 2011; 16: 109-30.

61. Karmieli-Miller O, Vu TR, Frankel RM, Holtman MC, Clyman SG, Hui SL, et al. Which experiences in the hidden curriculum teach students about professionalism? Acad Med 2011; 86: 369-77.

62. Taylor C, Farver C, Stoller JK. Can emotional intelligence training serve as an alternative approach to teaching professionalism to residents? Acad Med 2011; 86: 1551-4.

63. Twenge JM, Konrath S, Foster JD, Campbell WK, Bushman BJ. Egos inflating over time: a cross-temporal meta-analysis of the Narcissistic Personality Inventory. J Pers 2008; 76: 875-928. 